\title{
Graphs with four distinct Laplacian eigenvalues
}

\author{
A. Mohammadian • B. Tayfeh-Rezaie
}

Received: 4 April 2010 / Accepted: 5 April 2011 / Published online: 30 April 2011

(C) Springer Science+Business Media, LLC 2011

\begin{abstract}
In this paper, we investigate connected nonregular graphs with four distinct Laplacian eigenvalues. We characterize all such graphs which are bipartite or have exactly one multiple Laplacian eigenvalue. Other examples of interest are also presented.
\end{abstract}

Keywords Laplacian eigenvalue · Bipartite graph · Multiple eigenvalue

\section{Introduction}

Let $G$ be a graph with the vertex set $\left\{v_{1}, \ldots, v_{n}\right\}$. The adjacency matrix of $G$ is an $n \times n$ matrix $\mathcal{A}(G)$ whose $(i, j)$-entry is 1 if $v_{i}$ is adjacent to $v_{j}$ and is 0 otherwise. For any $i$, the degree of $v_{i}$, that is, the number of edges incident to $v_{i}$, is denoted by $d\left(v_{i}\right)$. The matrix $\mathcal{L}(G)=\mathcal{D}(G)-\mathcal{A}(G)$ is called the Laplacian matrix of $G$, where $\mathcal{D}(G)$ is the $n \times n$ diagonal matrix whose $(i, i)$-entry is $d\left(v_{i}\right)$. Since $\mathcal{A}(G)$ and $\mathcal{L}(G)$ are real symmetric matrices, their eigenvalues are real numbers. Moreover, $\mathcal{L}(G)$ is a positive semidefinite matrix with the smallest eigenvalue 0 . The eigenvalues of $\mathcal{A}(G)$ and $\mathcal{L}(G)$ are called the adjacency eigenvalues and the Laplacian eigenvalues of $G$, respectively.

Graphs with few distinct adjacency (Laplacian) eigenvalues form an interesting class of graphs and possess nice combinatorial properties. It is not hard to see that the number of distinct adjacency (Laplacian) eigenvalues of a connected graph is greater than the diameter of the graph. Therefore, all adjacency (Laplacian) eigenvalues of

A. Mohammadian · B. Tayfeh-Rezaie ( $₫)$

School of Mathematics, Institute for Research in Fundamental Sciences (IPM),

P.O. Box 19395-5746, Tehran, Iran

e-mail: tayfeh-r@ipm.ir

A. Mohammadian

e-mail: ali_m@ipm.ir 
a graph coincide if and only if the graph has no edge. Moreover, a graph has only two distinct adjacency (Laplacian) eigenvalues if and only if it is a disjoint union of complete graphs on the same number of vertices. It is well known that the class of all regular graphs with three distinct adjacency (Laplacian) eigenvalues coincides with the class of strongly regular graphs. For results on nonregular graphs with three adjacency eigenvalues, we refer the reader to $[1,3,5,16]$. Regular graphs with four adjacency (Laplacian) eigenvalues were studied in $[6,10]$, and nonregular bipartite graphs with four adjacency eigenvalues were investigated in [8,9], through the study of the incidence graphs of some combinatorial designs.

The authors of [7] showed that nonregular graphs with three Laplacian eigenvalues have nice structures like strongly regular graphs. More precisely, it is proved that in such a graph and also in its complement, any two nonadjacent vertices have the same number of common neighbors, which in turn yields that for degrees of vertices, there are only two possibilities. More results on these graphs can be found in [17]. In this paper, we study connected nonregular graphs with four distinct Laplacian eigenvalues. First, some examples are presented, and their Laplacian spectrum are computed. We then characterize all such graphs which are bipartite. Finally, we determine all instances of these graphs with exactly one multiple Laplacian eigenvalue.

\section{Notation and preliminaries}

Let us recall some definitions and notation to be used throughout the paper. For a graph $G$, the smallest degree of $G$ and the set of all neighbors of a vertex $v$ of $G$ are denoted by $\delta(G)$ and $\mathcal{N}(v)$, respectively. The complement of a graph $G$, denoted by $\bar{G}$, is the graph on the vertex set of $G$ such that two vertices of $\bar{G}$ are adjacent if and only if they are not adjacent in $G$. The union of two vertex disjoint graphs $G_{1}$ and $G_{2}$, denoted by $G_{1} \cup G_{2}$, is the graph whose vertex (respectively, edge) set is the union of vertex (respectively, edge) sets of $G_{1}$ and $G_{2}$. The join of two vertex disjoint graphs $G_{1}$ and $G_{2}$ is the graph obtained from $G_{1} \cup G_{2}$ by joining each vertex in $G_{1}$ with every vertex in $G_{2}$ and is denoted by $G_{1} \vee G_{2}$. We denote the complete graph on $n$ vertices and the complete bipartite graph with two parts of sizes $m$ and $n$ by $K_{n}$ and $K_{m, n}$, respectively. Also, the $n \times n$ identity matrix and the $m \times n$ all-one matrix will be denoted by $I_{n}$ and $J_{m \times n}$, respectively, and we will drop the subscripts whenever there is no danger of confusion.

Here, we recall some results from the literature that will be used in the subsequent sections.

Theorem 1 [15] Let $G$ and $H$ be two graphs with Laplacian spectra $\lambda_{1} \geqslant \cdots \geqslant \lambda_{n}$ and $\mu_{1} \geqslant \cdots \geqslant \mu_{m}$, respectively. Then the Laplacian spectra of $\bar{G}$ and $G \vee H$ are

$$
\begin{aligned}
& n-\lambda_{1}, \ldots, n-\lambda_{n-1}, 0 \text { and } \\
& n+m, m+\lambda_{1}, \ldots, m+\lambda_{n-1}, n+\mu_{1}, \ldots, n+\mu_{m-1}, 0,
\end{aligned}
$$

respectively. 
Theorem 2 [2, Theorem 1] Let $G$ be a graph with Laplacian spectrum $\lambda_{1} \geqslant \cdots \geqslant \lambda_{n}$ and with vertex degrees $d_{1} \geqslant \cdots \geqslant d_{n}$, and let $s \in\{1, \ldots, n\}$. Then $\lambda_{s} \geqslant d_{s}-s+2$, unless $G=K_{s} \cup(n-s) K_{1}$.

Theorem 3 [12, Theorem 2.1] The largest Laplacian eigenvalue of a graph $G$ is at most

$$
\max \{|\mathcal{N}(v) \cup \mathcal{N}(w)| \mid v \text { and } w \text { are two adjacent vertices of } G\} .
$$

Theorem 4 [13, Corollary 13.1.4] Let $G$ be a graph on $n$ vertices. Then $n$ is a Laplacian eigenvalue of $G$ if and only if $G$ is the join of two graphs.

Theorem 5 [14] Let $G$ be a graph which is not complete. Then the second smallest Laplacian eigenvalue of $G$ is at most $\delta(G)$. If the equality occurs, then $G$ is a join of a graph on $\delta(G)$ vertices with another graph.

\section{Examples}

In this section, we present some families of graphs with four distinct Laplacian eigenvalues and compute their Laplacian spectrum. Some of these examples are utilized in the characterizations given later.

Example 1 In [7], several examples and constructions of nonregular graphs with three distinct Laplacian eigenvalues are given. Let $G$ be a connected graph on $n$ vertices with three distinct Laplacian eigenvalues $0, \alpha, \beta$. Then the graphs $G \vee G$ and $G \vee K_{m}$ have four distinct Laplacian eigenvalues

$$
0, \alpha+n, \beta+n, 2 n \quad \text { and } \quad 0, \alpha+m, \beta+m, n+m,
$$

respectively.

Example 2 Let $n$ and $m$ be two distinct positive integers. By Theorem 1, it is easy to see that the Laplacian spectrum of $K_{n, m}$ is

$$
0^{[1]}, n^{[m-1]}, m^{[n-1]},(n+m)^{[1]} .
$$

Example 3 Let $n$ be a positive integer. If $\mathcal{M}$ is a matching in $K_{n}$ with $r$ edges, then the Laplacian spectrum of $K_{n} \backslash \mathcal{M}$ is

$$
0^{[1]},(n-2)^{[r]}, n^{[n-r-1]} .
$$

Thus, by Theorem 1, for any positive integer $m$, the Laplacian spectrum of $\left(K_{n} \backslash \mathcal{M}\right) \vee \bar{K}_{m}$ is

$$
0^{[1]}, n^{[m-1]},(n+m-2)^{[r]},(n+m)^{[n-r]} .
$$


Next, let $n \geqslant 2$, and let $G$ be a graph obtained by adding a matching with $r$ edges to $K_{n, n}$. Since $G$ is the complement of $\left(K_{n} \backslash \mathcal{M}_{1}\right) \cup\left(K_{n} \backslash \mathcal{M}_{2}\right)$ for two matchings $\mathcal{M}_{1}$ and $\mathcal{M}_{2}$, by Theorem 1, we conclude that the Laplacian spectrum of $G$ is

$$
0^{[1]}, n^{[2 n-r-2]},(n+2)^{[r]},(2 n)^{[1]} \text {. }
$$

Finally, let $n \geqslant 2$, and let $H$ be a graph obtained by adding a matching $\mathcal{M}$ with $r$ edges to $K_{1, n}$. Since $H$ is the complement of $K_{1} \cup\left(K_{n} \backslash \mathcal{M}\right)$, by Theorem 1, we find that the Laplacian spectrum of $H$ is

$$
0^{[1]}, 1^{[n-r-1]}, 3^{[r]},(n+1)^{[1]} \text {. }
$$

Example 4 Let $G_{1}$ and $G_{2}$ be two graphs on disjoint sets of $n_{1}$ and $n_{2}$ vertices, respectively. The corona $G_{1} \circ G_{2}$ of $G_{1}$ and $G_{2}$ is defined as the graph obtained by taking one copy of $G_{1}$ and $n_{1}$ copies of $G_{2}$, and then joining the $i$ th vertex of $G_{1}$ to every vertex in the $i$ th copy of $G_{2}$. For any positive integers $n$ and $m$, we have

$$
\begin{aligned}
\operatorname{det}\left(x I-\mathcal{L}\left(K_{n} \circ K_{m}\right)\right) & =\operatorname{det}\left[\begin{array}{c|c}
(x-n-m) I_{n}+J_{n} & I_{n} \otimes J_{1 \times m} \\
\hline I_{n} \otimes J_{m \times 1} & I_{n} \otimes\left((x-m-1) I_{m}+J_{m}\right)
\end{array}\right] \\
& =x(x-m-1)^{n(m-1)+1}\left(x^{2}-(n+m+1) x+n\right)^{n-1} .
\end{aligned}
$$

Therefore, $K_{n} \circ K_{m}$ has four distinct Laplacian eigenvalues that, along with their multiplicities, are

$$
0^{[1]},(m+1)^{[n(m-1)+1]},\left(\frac{(n+m+1) \pm \sqrt{(n+m+1)^{2}-4 n}}{2}\right)^{[n-1]} .
$$

Example 5 Let $D$ be the graph obtained from the incidence graph of a symmetric design with parameters $(v, k, \lambda)$ after joining any two vertices corresponding to the blocks of the design. We have

$$
\begin{aligned}
\operatorname{det}(x I-\mathcal{L}(D)) & =\operatorname{det}\left[\begin{array}{c|c}
(x-v-k) I_{v}+J_{v} & B \\
\hline B^{\top} & (x-k) I_{v}
\end{array}\right] \\
& =x(x-2 k)\left(x^{2}-(v+2 k) x+v(k+\lambda)\right)^{v-1},
\end{aligned}
$$

where $B$ is a $v \times v$ matrix such that $B B^{\top}=(k-\lambda) I+\lambda J$. Therefore, the Laplacian spectrum of $D$ is

$$
0^{[1]},(2 k)^{[1]},\left(\frac{v+2 k \pm \sqrt{v^{2}+4(k-\lambda)}}{2}\right)^{[v-1]} .
$$

Example 6 For any positive integers $r, s$, consider the vertex disjoint graphs $G_{1}, G_{2}, H_{1}, H_{2}$ such that $G_{1}, G_{2}$ are complete graphs on $r$ vertices and $H_{1}, H_{2}$ are graphs on $s$ vertices with no edges. We denote by $\mathcal{G}(r, s)$ the graph obtained by joining any vertex in subgraph $H_{1}$ of $G_{1} \vee H_{1}$ to any vertex in subgraph $H_{2}$ of $G_{2} \vee H_{2}$. 
Using Theorem 9.3.3 of [13] on equitable partitions, we find that the Laplacian spectrum of $G(r, s)$ is

$$
0^{[1]},(r+s)^{[2 r+2 s-3]},\left(\frac{3 s+r \pm \sqrt{(r+s)^{2}+4 r s}}{2}\right)^{[1]} .
$$

Example 7 Let $\mathcal{D}_{1}$ (respectively, $\mathcal{D}_{2}$ ) be the graph obtained from the incidence graph of the symmetric design with parameters $(7,3,1)$ (respectively, $(7,4,2))$ after joining a new vertex to all vertices corresponding to the blocks of the design. With an easy calculation, we obtain that $\mathcal{D}_{1}$ and $\mathcal{D}_{2}$ have the Laplacian spectra

$$
0^{[1]}, 2^{[6]}, 5^{[7]}, 9^{[1]} \text { and } 0^{[1]}, 3^{[6]}, 6^{[7]}, 10^{[1]},
$$

respectively.

\section{Bipartite graphs}

In this section, we characterize all connected bipartite graphs with four distinct Laplacian eigenvalues. It turns out that the bipartite graphs presented in Sect. 3 are in fact the only examples. In the following, we will use the notation associated to the graphs defined in Sect. 3.

Theorem 6 Let $G$ be a connected bipartite graph on $n \geqslant 5$ vertices. Then $G$ has four distinct Laplacian eigenvalues if and only if $G$ is either the incidence graph of a symmetric design or one of the graphs $\mathcal{D}_{1}, \mathcal{D}_{2}, \mathcal{G}(1,(n-2) / 2)$ and $K_{r, n-r}$, where $1<r<n / 2$.

Proof If $G$ is one of the graphs $\mathcal{D}_{1}, \mathcal{D}_{2}, \mathcal{G}(1,(n-2) / 2)$ and $K_{r, n-r}$ for some integer $1<r<n / 2$, then by the results of Sect. 3, $G$ has four distinct Laplacian eigenvalues. Also, if $G$ is the incidence graph of a symmetric design, by [4, p. 166] we are done. So assume that $G$ has four distinct Laplacian eigenvalues $0<\alpha<\beta<\gamma$. We suppose that $G$ is not a complete bipartite graph. By Theorem $5, \delta(G)>\alpha$. Assume that $\{A, B\}$ is the vertex partition of the bipartite graph $G$. Let

$$
\mathcal{A}(G)=\left[\begin{array}{cc}
0 & C \\
C^{\top} & 0
\end{array}\right] \text { and } P=\left[\begin{array}{cc}
-I_{r} & 0 \\
0 & I_{s}
\end{array}\right]
$$

for some $r \times s$ matrix $C$. Consider the matrix $\mathcal{Q}(G)=\mathcal{D}(G)+\mathcal{A}(G)$, the so-called signless Laplacian matrix of $G$. It is clear that $\mathcal{Q}(G)=P \mathcal{L}(G) P^{-1}$, and so $\mathcal{Q}(G)$ and $\mathcal{L}(G)$ have the same spectrum. Since $\mathcal{Q}(G)$ is a nonnegative irreducible matrix, by the Perron-Frobenius theorem [13, p. 178], the multiplicity of $\gamma$ is 1 , and there exists a positive eigenvector corresponding to $\gamma$. Since $x(x-\alpha)(x-\beta)(x-\gamma)$ is the minimal polynomial of $\mathcal{Q}(G)$, any row and any column of the matrix $\mathcal{Q}(G) \times$ $(\mathcal{Q}(G)-\alpha I)(\mathcal{Q}(G)-\beta I)$ is contained in the null space of $\mathcal{Q}(G)-\gamma I$, and so it is not hard to see that there exists a positive real eigenvector $v=\left(v_{1}, \ldots, v_{n}\right)$ corresponding to $\gamma$ such that

$$
\mathcal{Q}(G)(\mathcal{Q}(G)-\alpha I)(\mathcal{Q}(G)-\beta I)=v^{\top} v
$$


On the other hand, Lemma 2.1 of [17] shows that $(\mathcal{L}(G)-\alpha I)(\mathcal{L}(G)-\beta I) \times$ $(\mathcal{L}(G)-\gamma I)=-\frac{\alpha \beta \gamma}{n} J$, and so, multiplying both sides of (1) by $P$ from left and right, we find that

$$
(\mathcal{Q}(G)-\alpha I)(\mathcal{Q}(G)-\beta I)(\mathcal{Q}(G)-\gamma I)=\frac{\alpha \beta \gamma}{n}\left[\begin{array}{cc}
-J_{r} & J \\
J & -J_{s}
\end{array}\right] .
$$

Subtracting (2) from (1) gives

$$
\mathcal{Q}(G)^{2}-(\alpha+\beta) \mathcal{Q}(G)+\alpha \beta I=\frac{v^{\top} v}{\gamma}+\frac{\alpha \beta}{n}\left[\begin{array}{cc}
J_{r} & -J \\
-J & J_{s}
\end{array}\right] .
$$

Comparing the $(i, j)$-entries on both sides of (3), we will obtain the following:

$$
\left\{\begin{array}{l}
d(i)^{2}+(1-\alpha-\beta) d(i)=\frac{v_{i}^{2}}{\gamma}+\frac{\alpha \beta}{n}-\alpha \beta \quad \text { for all vertices } i \\
|\mathcal{N}(i) \cap \mathcal{N}(j)|=\frac{v_{i} v_{j}}{\gamma}+\frac{\alpha \beta}{n} \quad \text { if } i \text { and } j \text { are in the same part of } G \\
d(i)+d(j)=\frac{v_{i} v_{j}}{\gamma}+\alpha+\beta-\frac{\alpha \beta}{n} \quad \text { if } i \text { and } j \text { are adjacent; } \\
\frac{v_{i} v_{j}}{\gamma}=\frac{\alpha \beta}{n} \quad \text { if } i \text { and } j \text { are nonadjacent and in different parts. }
\end{array}\right.
$$

For any vertex $g$ of $G$, let $[g]$ denote the set of all vertices in the same part and with the same degree as of $g$. Using relations (4)-(7), we obtain the following facts about $G$.

Fact 1. For every two vertices $g$ and $g^{\prime}$ of $G$, if $d(g)=d\left(g^{\prime}\right)$, then $v_{g}=v_{g^{\prime}}$.

Fact 2. For every two vertices $a, a^{\prime} \in A$, if $v_{a}=v_{a^{\prime}}$, then $d(a)=d\left(a^{\prime}\right)$.

Fact 1 follows directly from (4), and in order to prove Fact 2, let $v_{a}=v_{a^{\prime}}$ for two vertices $a, a^{\prime} \in A$. By (5), there exists a vertex $b \in \mathcal{N}(a) \cap \mathcal{N}\left(a^{\prime}\right)$. Hence by (6), we have

$$
d(a)+d(b)=\frac{v_{a} v_{b}}{\gamma}+\alpha+\beta-\frac{\alpha \beta}{n}=\frac{v_{a^{\prime}} v_{b}}{\gamma}+\alpha+\beta-\frac{\alpha \beta}{n}=d\left(a^{\prime}\right)+d(b),
$$

and the assertion follows.

Fact 3. For every two vertices $a, a^{\prime} \in A$ of the same degree, all vertices in $B \backslash$ $\left(\mathcal{N}(a) \cap \mathcal{N}\left(a^{\prime}\right)\right)$ have the same degree.

Fact 4 . For every two nonadjacent vertices $a \in A$ and $b \in B$, every vertex in $[a]$ (respectively, $[b]$ ) is adjacent to every vertex in $B \backslash[b]$ (respectively, $A \backslash[a]$ ).

The above two facts follow from (7) and Facts 1 and 2. Notice that Facts 2 and 3 also hold for vertices in part $B$.

Fact 5. For every vertices $a, a^{\prime} \in A$ and $b, b^{\prime} \in B$, if the induced subgraph on $\left\{a, a^{\prime}, b, b^{\prime}\right\}$ is $K_{2,2}$, then either $d(a)=d\left(a^{\prime}\right)$ or $d(b)=d\left(b^{\prime}\right)$.

In order to prove Fact 5, note that by applying (6) for vertices $a, a^{\prime} \in A$ and $b$, $b^{\prime} \in B$, we easily deduce that

$$
d(a)-d\left(a^{\prime}\right)=\frac{\left(v_{a}-v_{a^{\prime}}\right) v_{b}}{\gamma} \quad \text { and } \quad d(a)-d\left(a^{\prime}\right)=\frac{\left(v_{a}-v_{a^{\prime}}\right) v_{b^{\prime}}}{\gamma} .
$$


Hence, if $d(a) \neq d\left(a^{\prime}\right)$, then $v_{b}=v_{b^{\prime}}$, and therefore from Fact 2 it follows that $d(b)=d\left(b^{\prime}\right)$, as desired. Now we are able to continue the proof of the theorem.

First, we assume that there exist two nonadjacent vertices $a \in A$ and $b \in B$ with at least one edge between $[a]$ and $[b]$. By Facts 4 and 5, there is no edge between $A \backslash[a]$ and $B \backslash[b]$. This, along with Fact 4, shows that for any vertex $a^{\prime} \in A \backslash$ $[a]$ and $b^{\prime} \in B \backslash[b]$, we have $\mathcal{N}\left(a^{\prime}\right)=[b]$ and $\mathcal{N}\left(b^{\prime}\right)=[a]$. Therefore, applying (5) for any two vertices in $[a]$ and any two vertices in $[b]$ and then Fisher's inequality [13, Lemma 5.10.1], it turns out that the induced subgraph of $G$ on $[a] \cup[b]$ is the incidence graph of a symmetric design with parameters, say $(m, k, \lambda)$. So $m=|[a]|=$ $|[b]|$.

We claim that either $[a]=A$ or $[b]=B$. By contrary, assume that there are two vertices $a^{\prime} \in A \backslash[a]$ and $b^{\prime} \in B \backslash[b]$. Then, by Fact 1 , we have $v_{a^{\prime}}=v_{b^{\prime}}$. By (5), we obtain that

$$
\frac{v_{a} v_{a^{\prime}}}{\gamma}+\frac{\alpha \beta}{n}=\left|\mathcal{N}(a) \cap \mathcal{N}\left(a^{\prime}\right)\right|=\lambda=\left|\mathcal{N}(b) \cap \mathcal{N}\left(b^{\prime}\right)\right|=\frac{v_{b} v_{b^{\prime}}}{\gamma}+\frac{\alpha \beta}{n},
$$

and thus $v_{a}=v_{b}$. Now, applying (7), we find that

$$
\frac{v_{a}^{2}}{\gamma}=\frac{\alpha \beta}{n}=\frac{v_{a^{\prime}}^{2}}{\gamma}
$$

and so $v_{a}=v_{a^{\prime}}$, a contradiction. Hence, without loss of generality, we may suppose that $[a]=A$. Let $|B \backslash[b]|=r$. If $r=0$, then $G$ is the incidence graph of a symmetric design. So assume that $r \geqslant 1$. We have

$$
\mathcal{Q}(G)=\left[\begin{array}{c|c|c}
(r+k) I_{m} & C & J \\
\hline C^{\top} & k I_{m} & 0 \\
\hline J & 0 & m I_{r}
\end{array}\right],
$$

for some $m \times m$ matrix $C$ in which $C C^{\top}=(k-\lambda) I+\lambda J$. An easy calculation shows that $\operatorname{det}(x I-\mathcal{Q}(G))=x(x-m)^{r-1}\left(p_{1}(x)\right)^{m-1} p_{2}(x)$, where $p_{1}(x)=x^{2}-$ $(r+2 k) x+r k+m \lambda$ and $p_{2}(x)=x^{2}-(r+2 k+m) x+k(r+2 m)$. It is straightforward to verify that the polynomials $p_{1}(x)$ and $p_{2}(x)$ have no multiple root, and so they must have a common root. Considering simultaneously the equations $p_{1}(x)=0$ and $p_{2}(x)=0$, we find that the common root of $p_{1}(x)$ and $p_{2}(x)$ is $x_{0}=2 k-\lambda$ and the other roots of $p_{1}(x)$ and $p_{2}(x)$ are $x_{1}=r+\lambda$ and $x_{2}=r+\lambda+m$, respectively. From $p_{1}\left(x_{0}\right)=0$, we deduce that $m=\left(k^{2}-r-1\right) /(k-r-1)$. One can easily check that $m \notin\left\{x_{0}, x_{1}, x_{2}\right\}$ and so $r=1$, since $G$ has four distinct Laplacian eigenvalues. Thus, $m=k+2+2 /(k-2)$ and since $m$ is an integer, we conclude that either $k=3$ or $k=4$. Using the fact $\lambda(m-1)=k(k-1)$ from design theory, in the first case, we find that $G=\mathcal{D}_{1}$ and in the latter case, $G=\mathcal{D}_{2}$.

Next, suppose that for every two nonadjacent vertices $a \in A$ and $b \in B$, there is no edge between $[a]$ and $[b]$. Using Facts 4 and 5, we consider the following two cases.

Case 1. There are two vertices $x \in A$ and $y \in B$ such that $\mathcal{N}(x)=B$ and $\mathcal{N}(y)=A$. By Fact 5, there is no edge from $A \backslash[x]$ to $B \backslash[y]$. We want to establish that $|A \backslash[x]|=|B \backslash[y]|=1$. By contrary and with no loss of generality, assume 
that there are two vertices $x^{\prime}, x^{\prime \prime} \in A \backslash[x]$. By (5), we have

$$
\frac{v_{x} v_{x^{\prime \prime}}}{\gamma}+\frac{\alpha \beta}{n}=|[y]|=\frac{v_{x^{\prime}} v_{x^{\prime \prime}}}{\gamma}+\frac{\alpha \beta}{n} .
$$

This yields that $v_{x}=v_{x^{\prime}}$, which contradicts Fact 2. Therefore, $G=K_{r, s}-e$. We want to show that $r=s$. To continue the proof, we need the following fact.

Fact 6. For every two distinct vertices $g$ and $g^{\prime}$ of $G$, if $\mathcal{N}(g)=\mathcal{N}\left(g^{\prime}\right)$, then $d(g)=$ $d\left(g^{\prime}\right)=\beta$.

In order to establish Fact 6 , let $\mathcal{N}(g)=\mathcal{N}\left(g^{\prime}\right)$ for two distinct vertices $g$ and $g^{\prime}$ of $G$. Using (5), we have $d(g)=v_{g} v_{g^{\prime}} / \gamma+\alpha \beta / n$, and applying (4), we find that $d(g)^{2}+(1-\alpha-\beta) d(g)=d(g)-\alpha \beta$. From Theorem 5, $\delta(G)>\alpha$, and therefore $d(g)=d\left(g^{\prime}\right)=\beta$.

If $|[x]| \geqslant 2$ and $|[y]| \geqslant 2$, then applying Fact 6 , we find that $G=K_{n / 2, n / 2}-e=$ $\mathcal{G}(1,(n-2) / 2)$, and we are done. Suppose without loss of generality that $|[x]| \geqslant 2$ and $|[y]|=1$. Hence Fact 6 yields that $d(x)=\beta=2$, and so applying Theorem 2 for $s=2$, we deduce that $n-3=|[x]|=d(z) \leqslant \beta=2$ for the vertex $z \in B \backslash[y]$. From $n \geqslant 5$ we deduce that $G=K_{2,3}-e$, which is a contradiction, since $G$ has just four distinct Laplacian eigenvalues.

Case 2. Without loss of generality, assume that there is no vertex such $a \in A$ that $\mathcal{N}(a)=B$. Consider two nonadjacent vertices $a_{1} \in A$ and $b_{1} \in B$. Since $G$ is connected and there is no edge between $\left[a_{1}\right]$ and $\left[b_{1}\right]$, by the assumption of Case 2 and Fact 4, there exist two nonadjacent vertices $a_{2} \in A \backslash\left[a_{1}\right]$ and $b_{2} \in B \backslash\left[b_{1}\right]$. Now by the assumption of Case 2 , there is no edge between $\left[a_{2}\right]$ and $\left[b_{2}\right]$, and so by Fact 4 , the subgraphs of $G$ induced on $\left[a_{1}\right] \cup\left[b_{2}\right]$ and $\left[a_{2}\right] \cup\left[b_{1}\right]$ are complete bipartite. By (5), $\left|\mathcal{N}\left(b_{1}\right) \cap \mathcal{N}\left(b_{2}\right)\right| \neq 0$, and so there is a vertex $a_{3} \in A \backslash\left(\left[a_{1}\right] \cup\left[a_{2}\right]\right)$. By the assumption of Case 2 and Fact 4, there exists a vertex $b_{3} \in B \backslash\left(\left[b_{1}\right] \cup\left[b_{2}\right]\right)$ such that $a_{3}$ and $b_{3}$ are nonadjacent. Now, if there is a vertex $x \in A \backslash\left(\left[a_{1}\right] \cup\left[a_{2}\right] \cup\left[a_{3}\right]\right)$, then we get a contradiction by Fact 5 . Hence $A=\left[a_{1}\right] \cup\left[a_{2}\right] \cup\left[a_{3}\right]$ and similarly $B=\left[b_{1}\right] \cup\left[b_{2}\right] \cup\left[b_{3}\right]$. If two of the sets $\left[a_{1}\right],\left[a_{2}\right],\left[a_{3}\right]$ have only one vertex, say $\left[a_{1}\right]$ and $\left[a_{2}\right]$, then $d\left(b_{1}\right)=d\left(b_{2}\right)$, which is impossible. Therefore, using Fact 6 , the degree of at least two vertices of $a_{1}, a_{2}, a_{3}$ are $\beta$, which is again impossible. This completes the proof.

We remark that, as well known, for bipartite graphs Laplacian and signless Laplacian are similar matrices. Hence Theorem 6 also holds for signless Laplacian.

\section{Multiple Laplacian eigenvalues}

All graphs with three distinct Laplacian eigenvalues such that exactly one of them is multiple are previously identified.

Theorem 7 [11, Theorems 2.8 and 2.9] Let $G$ be a graph on $n \geqslant 3$ vertices whose distinct Laplacian eigenvalues are $0<\alpha<\beta$. Then the following hold.

(i) The multiplicity of $\alpha$ is $n-2$ if and only if $G$ is one of the graphs $K_{n / 2, n / 2}$ or $K_{1, n-1}$. 
(ii) The multiplicity of $\beta$ is $n-2$ if and only if $G$ is the graph $K_{n}-e$.

In this section, we characterize all graphs with four distinct Laplacian eigenvalues such that exactly one of their Laplacian eigenvalues is multiple. Notice that we will use the notation associated to the graphs defined in Sect. 3.

Theorem 8 Let $G$ be a graph on $n \geqslant 5$ vertices whose distinct Laplacian eigenvalues are $0<\alpha<\beta<\gamma$. Then the multiplicity of $\alpha$ is $n-3$ if and only if $G$ is one of the graphs $K_{2, n-2}, K_{n / 2, n / 2}+$ e or $K_{1, n-1}+e$.

Proof If $G$ is one of the graphs $K_{2, n-2}, K_{n / 2, n / 2}+e$ or $K_{1, n}+e$, then we are done by the results of Sect. 3. For the converse, assume that $\alpha$ is the only multiple Laplacian eigenvalue of $G$. This implies that $\alpha$ is an integer. Let $d_{1} \geqslant \cdots \geqslant d_{n}$ be the vertex degrees of $G$. By Theorem $2, d_{3} \leqslant \alpha+1$. Also, Theorem 5 yields that $d_{n} \geqslant \alpha$. It follows that $d_{3}, \ldots, d_{n} \in\{\alpha, \alpha+1\}$.

First, assume that $d_{n}=\alpha+1$. Since the trace of $\mathcal{L}(G)$ is equal to the sum of all vertex degrees of $G$, we have $(n-3) \alpha+\beta+\gamma=d_{1}+d_{2}+(n-2)(\alpha+1)$. Hence, $\beta+\gamma=d_{1}+d_{2}+\alpha+n-2$. By Theorem $3, \gamma \leqslant \min \left\{n, d_{1}+d_{2}\right\}$, and so $n+d_{1}+d_{2} \geqslant 2 \gamma>\beta+\gamma=d_{1}+d_{2}+\alpha+n-2$, which yields that $\alpha=1$ and therefore $\gamma=n=d_{1}+d_{2}$. By Theorem 4, $G=H_{1} \vee H_{2}$ for some graphs $H_{1}$ and $H_{2}$. Applying Theorem 1, we conclude that either $H_{1}$ or $H_{2}$ has one vertex. This shows that $d_{1}=n-1$, which contradicts $d_{1}+d_{2}=n$ and $d_{2} \geqslant 2$.

Next, suppose that $d_{n}=\alpha$. By Theorem 5, $G$ has the form $H_{1} \vee H_{2}$ for some graphs $H_{1}$ and $H_{2}$, where $H_{1}$ has $\alpha$ vertices. For any nonzero Laplacian eigenvalue $\mu$ of $H_{2}$, we must have $\beta=\mu+\alpha$, so $H_{2}$ has at most one nonzero Laplacian eigenvalue. Therefore, $H_{2}$ has at most one edge. Let $\lambda_{1} \geqslant \cdots \geqslant \lambda_{\alpha}$ be the Laplacian eigenvalues of $H_{1}$.

Assume that $H_{2}$ has no edges. If $\alpha \leqslant 2$, then it is straightforward to see that $G=$ $K_{2, n-2}$. So suppose that $\alpha \geqslant 3$. Using Theorem 1 , we find that $\beta=\lambda_{1}+n-\alpha$ and $\alpha=\lambda_{2}+n-\alpha=\cdots=\lambda_{\alpha-1}+n-\alpha$. If $H_{1}$ is connected, then Theorem 7(i) implies that $H_{1}$ is the join of two graphs, and so using Theorems 1 and 4, the multiplicity of $\gamma$ is at least 2, a contradiction. Thus $H_{1}$ is not connected, and so $\lambda_{2}=\cdots=\lambda_{\alpha-1}=0$. Thus $\alpha=n-\alpha$, and since $\beta>\alpha, H_{1}$ has one nonzero Laplacian eigenvalue. This implies that $G=K_{n / 2, n / 2}+e$.

Now assume that $H_{2}$ has one edge. If $\alpha=1$, then we have $G=K_{1, n}+e$. So suppose that $\alpha \geqslant 2$. Using Theorem 1 , we find that $\beta=\alpha+2$ and $\alpha=\lambda_{1}+n-\alpha=$ $\cdots=\lambda_{\alpha-1}+n-\alpha$. Hence either $H_{1}=K_{\alpha}$ or $H_{1}=\bar{K}_{\alpha}$. Since the multiplicity of $\gamma$ is 1 , Theorem 1 implies that the first case is impossible, and in the latter case we again find that $G=K_{n / 2, n / 2}+e$.

Theorem 9 Let $G$ be a graph on $n \geqslant 5$ vertices whose distinct Laplacian eigenvalues are $0<\alpha<\beta<\gamma$. Then the multiplicity of $\gamma$ is $n-3$ if and only if $G=K_{n-3} \vee \bar{K}_{1,2}$.

Proof First assume that the multiplicity of $\gamma$ is $n-3$. If $\gamma \neq n$, then by Theorem 1 , the Laplacian spectrum of $\bar{G}$ is

$$
0^{[1]},(n-\gamma)^{[n-3]},(n-\beta)^{[1]},(n-\alpha)^{[1]} .
$$


Since $n-\alpha \neq n$, we obtain a contradiction using Theorem 8. Hence $\gamma=n$, and therefore by Theorem 1, the Laplacian spectrum of $\bar{G}$ is

$$
0^{[n-2]},(n-\beta)^{[1]},(n-\alpha)^{[1]} \text {. }
$$

It follows that $\bar{G}$ has $n-2$ connected components, so $\bar{G}=\bar{K}_{n-3} \cup K_{1,2}$, and the assertion follows. Notice that the converse is clear, since the Laplacian spectrum of $K_{n-3} \vee \bar{K}_{1,2}$ is

$$
0^{[1]},(n-3)^{[1]},(n-1)^{[1]}, n^{[n-3]} \text {. }
$$

Theorem 10 Let $G$ be a graph on $n \geqslant 5$ vertices whose distinct Laplacian eigenvalues are $0<\alpha<\beta<\gamma$. Then the multiplicity of $\beta$ is $n-3$ if and only if $G$ is one of the graphs $K_{1} \vee 2 K_{(n-1) / 2}, \bar{K}_{n / 3} \vee 2 K_{n / 3}, K_{n-1}+e$, or $\mathcal{G}(r, n-r)$ for some $r$.

Proof If $G$ is one of the graphs $K_{1} \vee 2 K_{(n-1) / 2}, \bar{K}_{n / 3} \vee 2 K_{n / 3}, K_{n-1}+e$, or $\mathcal{G}(r, n-r)$ for some $1 \leqslant r \leqslant n-1$, then the assertion easily follows from the results of Sect. 3 and Theorem 1. For the converse, assume that the multiplicity of $\beta$ is $n-3$.

First, suppose that $\gamma \neq n$. Using Theorem 4, this means that $G$ is not a join of two graphs. Since $\beta$ is the only multiple Laplacian eigenvalue of $G, \beta$ is an integer. Let $\left\{v_{1}, \ldots, v_{n}\right\}$ and $d_{1} \geqslant \cdots \geqslant d_{n}$ be the vertex set and the vertex degrees of $G$, respectively. For simplicity, we let $d\left(v_{i}\right)=d_{i}$ for any $i$. By Theorem $2, d_{2} \leqslant \beta$, and also by Theorem 1 , we have $n-1-d_{n-1} \leqslant n-\beta$. It follows that $d_{2}, \ldots, d_{n-1} \in$ $\{\beta, \beta-1\}$. Define

$$
U_{1}=\left(\left\{v_{1}\right\} \cup\left\{v_{i} \mid d_{i}=\beta\right\}\right) \backslash\left\{v_{n}\right\} \quad \text { and } \quad U_{2}=\left(\left\{v_{n}\right\} \cup\left\{v_{i} \mid d_{i}=\beta-1\right\}\right) \backslash\left\{v_{1}\right\} .
$$

Since the multiplicity of $\beta$ is $n-3$, every $4 \times 4$ principal submatrix of $\beta I-\mathcal{L}(G)$ is singular. We frequently use this property in what follows.

We prove that $\mathcal{N}(u)=\mathcal{N}(v)$ for any two nonadjacent vertices $u, v \in U_{1}$. Suppose otherwise that there exist two nonadjacent vertices $x, y \in U_{1}$ such that $d(y)=\beta$ and $\mathcal{N}(x) \backslash \mathcal{N}(y)$ contains at least a vertex $z$ with $d(z) \leqslant \beta$. For a vertex $t \in \mathcal{N}(y)$, the principal submatrix of $\beta I-\mathcal{L}(G)$ corresponding to the vertices $x, y, z, t$ is

$$
\left[\begin{array}{cccc}
\beta-d(x) & 0 & 1 & \xi_{1} \\
0 & 0 & 0 & 1 \\
1 & 0 & \beta-d(z) & \xi_{2} \\
\xi_{1} & 1 & \xi_{2} & \beta-d(t)
\end{array}\right]
$$

for some $\xi_{1}, \xi_{2} \in\{0,1\}$. Its determinant is $1+(d(x)-\beta)(\beta-d(z)) \neq 0$, since $d(z) \leqslant$ $\beta \leqslant d(x)$. Note that the Laplacian spectrum of $\bar{G}$ has the form similar to that of $G$, so applying the above property for $\bar{G}$, we conclude that $\mathcal{N}(u) \backslash\{v\}=\mathcal{N}(v) \backslash\{u\}$ for any two adjacent vertices $u, v \in U_{2}$.

Denote the induced subgraphs of $G$ on $U_{1}$ and $U_{2}$ by $G_{1}$ and $G_{2}$, respectively. The above properties on $U_{1}$ and $U_{2}$ show that any two nonadjacent vertices of each of the subgraphs $G_{1}$ in $G$ and $\overline{G_{2}}$ in $\bar{G}$ have the same neighborhoods. By a graph theoretic argument, it is easy to see that a graph with this property must be a complete multipartite graph. Therefore, $G_{1}$ is a complete multipartite graph, and $G_{2}$ is a disjoint 
union of complete graphs. By contradiction, suppose that there are three independent vertices in $U_{2}$, say $x, y, z$. Since the multiplicity of 0 in the Laplacian spectrum of any graph is equal to the number of its connected components [13, Lemma 13.1.1], $G$ is connected, and without loss of generality, we may assume that $x$ is adjacent to a vertex $t \in U_{1}$ and $d(y)=d(z)=\beta-1$. Now, the principal submatrix of $\beta I-\mathcal{L}(G)$ corresponding to the vertices $x, y, z, t$ is

$$
\left[\begin{array}{cccc}
\beta-d(x) & 0 & 0 & 1 \\
0 & 1 & 0 & \xi_{1} \\
0 & 0 & 1 & \xi_{2} \\
1 & \xi_{1} & \xi_{2} & \beta-d(t)
\end{array}\right]
$$

for some $\xi_{1}, \xi_{2} \in\{0,1\}$, whose determinant is $(d(x)-\beta)\left(\xi_{1}+\xi_{2}-(\beta-d(t))\right.$ $-1 \neq 0$, a contradiction. Hence $G_{2}$ is a disjoint union of at most two complete graphs. If $G_{2}$ is a complete graph, then $G$ would be a join of two graphs, a contradiction. Thus $G_{2}$ is a disjoint union of two complete graphs, and by considering $\bar{G}$ instead of $G$, we conclude that $G_{1}$ is a complete bipartite graph. Let $\left\{V_{1}, V_{2}\right\}$ be the vertex partition of the bipartite graph $G_{1}$, and $W_{1}, W_{2}$ be the vertex sets of two cliques of $G_{2}$. Since $G$ is a connected graph which is not a join of two graphs, without loss of generality, we may assume that any vertex in $V_{i}$ is adjacent to any vertex in $W_{i}$ for $i=1,2$ and that $G$ has no further edges. For $i=1,2$, let $a_{i} \in V_{i}$ and $b_{i} \in W_{i}$. We have $\min \left\{d\left(a_{1}\right), d\left(a_{2}\right)\right\} \geqslant \max \left\{d\left(b_{1}\right), d\left(b_{2}\right)\right\}+1$. This yields that $\left|V_{1}\right|=\left|V_{2}\right|$ and $\left|W_{1}\right|=\left|W_{2}\right|$, and therefore $G=\mathcal{G}(r, s)$ for some $r, s$.

Next, suppose that $\gamma=n$. By Theorem 1 , the Laplacian spectrum of $\bar{G}$ is

$$
0^{[2]},(n-\beta)^{[n-3]},(n-\alpha)^{[1]} \text {. }
$$

Hence $\bar{G}$ has two connected components, say $H_{1}$ and $H_{2}$. Since the Laplacian spectrum of $K_{m}$ is $0^{[1]}, m^{[m-1]}$, it is easily checked that the distinct Laplacian eigenvalues of one of graphs $H_{1}$ and $H_{2}$, say $H_{1}$, are $0<n-\beta<n-\alpha$, and $H_{2}$ is either a single vertex or a complete graph on $n-\beta$ vertices. By Theorem 7(i), $H_{1}$ is either $K_{1, s}$ for some $s$ or a regular complete bipartite graph. It follows that $G$ is one of the graphs $K_{1} \vee 2 K_{(n-1) / 2}, \bar{K}_{n / 3} \vee 2 K_{n / 3}$, or $K_{n-1}+e$.

Acknowledgements The authors gratefully thank one of the anonymous referees for her/his valuable comments. The research of the first author was in part supported by a grant from IPM.

\section{References}

1. Bridges, W.G., Mena, R.A.: Multiplicative cones—a family of three eigenvalue graphs. Aequ. Math. 22, 208-214 (1981)

2. Brouwer, A.E., Haemers, W.H.: A lower bound for the Laplacian eigenvalues of a graph-proof of a conjecture by Guo. Linear Algebra Appl. 429, 2131-2135 (2008)

3. de Caen, D., van Dam, E.R., Spence, E.: A nonregular analogue of conference graphs. J. Comb. Theory, Ser. A 88, 194-204 (1999)

4. Cvetković, D.M., Doob, M., Sachs, H.: Spectra of Graphs: Theory and Application. V.E.B. Deutscher Verlag der Wissenschaften, Berlin (1979)

5. van Dam, E.R.: Nonregular graphs with three eigenvalues. J. Comb. Theory, Ser. B 73, 101-118 (1998) 
6. van Dam, E.R.: Regular graphs with four eigenvalues. Linear Algebra Appl. 226/228, 139-162 (1995)

7. van Dam, E.R., Haemers, W.H.: Graphs with constant $\mu$ and $\bar{\mu}$. Discrete Math. 182, 293-307 (1998)

8. van Dam, E.R., Spence, E.: Combinatorial designs with two singular values II. Partial geometric designs. Linear Algebra Appl. 396, 303-316 (2005)

9. van Dam, E.R., Spence, E.: Combinatorial designs with two singular values I: uniform multiplicative designs. J. Comb. Theory, Ser. A 107, 127-142 (2004)

10. van Dam, E.R., Spence, E.: Small regular graphs with four eigenvalues. Discrete Math. 189, 233-257 (1998)

11. Das, K.C.: A sharp upper bound for the number of spanning trees of a graph. Graphs Comb. 23, 625-632 (2007)

12. Das, K.C.: An improved upper bound for Laplacian graph eigenvalues. Linear Algebra Appl. 368, 269-278 (2003)

13. Godsil, C., Royle, G.: Algebraic Graph Theory. Springer, New York (2001)

14. Kirkland, S.J., Molitierno, J.J., Neumann, M., Shader, B.L.: On graphs with equal algebraic and vertex connectivity. Linear Algebra Appl. 341, 45-56 (2002)

15. Merris, R.: Laplacian graph eigenvectors. Linear Algebra Appl. 278, 221-236 (1998)

16. Muzychuk, M., Klin, M.: On graphs with three eigenvalues. Discrete Math. 189, 191-207 (1998)

17. Wang, Y., Fan, Y., Tan, Y.: On graphs with three distinct Laplacian eigenvalues. Appl. Math. J. Chin. Univ. Ser. B 22, 478-484 (2007) 